tremendous waste of energy if the young man has to learn undirected, has to discover known facts, has to do research work unencouraged. All that we professional people all over the world. have learned is not our own private possession, but a great privilege which has been entrusted to us, and it is our duty to pass on this loan, our knowledge, enlarged by the result of our own research work, to the rising generation. If we betray this trust we provoke hatred where love and gratitude ought to be, and lose our only right to exist. Disappointed youth will fight against old age, and youth will prove the stronger.

There is still time for reforms all over the world. They are urgent everywhere, as much of our schooling has been inherited from a prescientific age (T. A. Lauwery, 1941). But fate knocks at the door. Let us older ones listen to the fateful sign; otherwise we will sink back to the moral standard of prehistoric wild tribes where youth killed the aged as a useless, bothersome burden. We are servants of the future smoothing the path of youth.

\title{
GLASS IN THE ANTERIOR CHAMBER
}

BY

\section{T. Collyer Summers and Henry Hobbs \\ OLDCHURCH COUNTY HOSPITAL, ROMFORD, ESSEX}

THE subject of glass in the anterior chamber of the eye assumes added importance at the present time from the frequency of eye injuries due to glass among air raid casualties; and the treatment of such casualties is therefore a matter of common interest. Present practice seems to be based on the belief that glass retained in the anterior chamber spells the doom of the eye; one may quote, for example, a recent annotation in the Brit. Jl. of Ophthal., ". . . . eyes containing glass fragments shrink relatively quickly, save in a few exceptional cases when a glass fragment has remained in the vitreous for years without complaint."'1 Nevertheless, cases $(2,3,4)$ are to be found in the literature (Bickerton's, as far back as 1888), in which such fragments have remained in the anterior chamber for long periods and a useful eye has been retained after their removal.

The purpose of this paper is to report a case in which a spicule of glass was necessarily retained for some months in the anterior chamber, subsequently giving rise to severe irritation and almost total loss of vision, with recovery of full visual acuity after its removal. 
The patient, C.W., aged 33 years, was involved in an air raid on September 23, 1940, sustaining multiple injuries. The right eye was cut to pieces and immediate excision was necessary. The left eye was stated to have a piece of glass embedded in it, and on examination a triangular flap wound of the cornea was seen, in which was lodged a small spicule of glass, size approximately $3 \times 1 \times 1 \mathrm{~mm}$. This was no sooner located than it was seen to slide over the posterior lip of the wound and fall into the inferior angle of the anterior chamber.

As it was impossible to use a keratome to make a fresh wound and equally impossible to remove the glass through the valvular incision, it was decided to await healing of the wound and then attempt removal of the foreign body. Rapid healing took place, but cultures for weeks showed a pure growth of staphylococcus aureus, despite irrigation with merthiolate $1 / 10,000$. Vision at this time was excellent and on January 4, 1941, the patient was discharged under outpatient supervision.

On February 28, 1941, the patient was readmitted to Oldchurch County Hospital, complaining of sudden, severe irritation of the eye with greatly reduced vision - at that time perception of shadows only. On examination, the lower cornea showed a dense interstitial haze and, above, considerable bedewing of the endothelium; ciliary injection was marked. Treatment was begun with atropine, Maddox Heater and daily fifteen minute treatments with ultrashort wave diathermy with the result that the corneal condition rapidly cleared and on March 7 the visual acuity, unaided, was $6 / 24$. There was still, however, a great deal of opacity in the lower part of the cornea and no foreign body could be seen.

With continued treatment the corneal condition improved further and on March 31 the cornea was clear enough to enable the spicule of glass to be seen with great difficulty, lying in the extreme lower angle of the anterior chamber. The vision at this time with a small correction was $6 / 12$.

Four successive sterile cultures having been obtained, on April 4 removal of the glass spicule was decided on, and inferior hemisection of the cornea was performed. It was intended to perform iridectomy of the root of the iris in the hope that the glass would be removed with the iris, but on inserting the blades of Barraquer's forceps, the glass was immediately felt and removed without further manipulation.

The wound healed rapidly and the patient now has a visual acuity of $6 / 5$ with a small correction.

Our reasons for reporting the case are threefold :-

1. To emphasise the severity of the irritation caused by glass in the anterior chamber. 
2. To confirm the possibility of its removal once it has been located.

3. To record that full recovery of vision is possible after removal.

\title{
REFERENCES
}

1. Brit. Jl. Ophthal., Annotation, April, 1941.

2. Bickerton.-Brit. Med. Jl., Vol. I, 1888.

3. Claiborne.-Amer. Jl. of Surgery, Vol. XXXVI, 1922.

4. BURKe.-Trans. Amer. Ophthal. Soc., Vol. XXVIII, pp. 53-60, 1930.

\section{THE AETIOLOGY OF AN OBSCURE CASE OF RETROBULBAR NEURITIS}

BY

\author{
ROSA FORD \\ LONDON
}

WE still see a number of cases of retrobulbar neuritis the cause of which is obscure. Carroll, ${ }^{1}$ in 1940 , went through 100 of his cases and found the cause undetermined in no fewer than 34 .

When we have excluded trauma and tumour, the Wassermann reaction is negative, and a negative report is received from an investigation of the para-nasal sinuses, we are left with the suspicion that the condition may be the first symptom of disseminated sclerosis. As we do not know the cause of this either, the position is an unsatisfactory one.

The case here described was at first in this unexplained category, but later events cleared the diagnosis, so that a report may help to throw light on other obscure cases.

A.E., a woman aged 31 years, was first seen in April, 1930, because for 2 months she had been unable to do the fine needlework at which, till then, she had been particularly good. Vision was $6 / 18$ in each eye and the fundi were normal.

In the search for a cause, every investigation, medical, dental (with X-ray), rhinological (with X-ray) and the Wassermann reaction proved negative. During the next few months her sight varied, occasionally becoming $6 / 9$, but in July it was still only $6 / 18$ in both eyes.

Seven months from the onset, it was decided to explore the sphenoidal sinuses, and thick pus was withdrawn from the left and cloudy mucus from the right. Recovery followed when both spheno-ethmoidal sinuses were opened, but this was speedily 